This document is the Accepted Manuscript version of a Published Work that appeared in final form in Journal of Physical

Chemistry C, copyright (c) American Chemical Society after peer review and technical editing by the publisher. To access the final edited and published work see:

https://dx.doi.org/10.1021/acs.jpcc.7b09147. 


\title{
Electronic Structure Of Titanylphthalocyanine Layers On Ag(111)
}

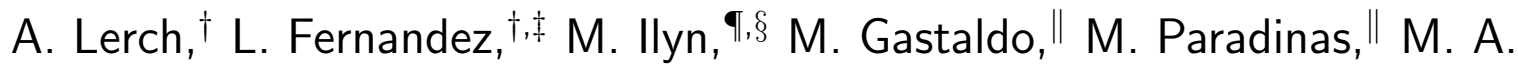

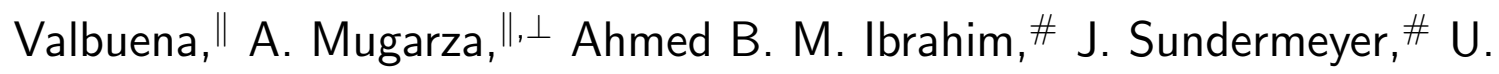 \\ Höfer, ${ }^{\dagger}$ and F. Schiller*,†,
}

Fachbereich Physik und Zentrum für Materialwissenschaften, Philipps-Universität Marburg, 35032 Marburg, Germany, Institut für Festkörperphysik, TU Dresden, D-01062 Dresden, Germany, Centro de Física de Materiales (CSIC-UPV-EHU) and Materials Physics Center (MPC), 20018 San Sebastián, Spain, Donostia International Physics Center, 20018 Donostia-San Sebastián, Spain, Catalan Institute of Nanoscience and Nanotechnology (ICN2), CSIC and The Barcelona Institute of Science and Technology, Campus UAB, Bellaterra, 08193 Barcelona, Spain, ICREA, Institució Catalana de Recerca i Estudis Avançats, Lluis Companys 23, 08010 Barcelona, Spain, and Fachbereich Chemie, Philipps-Universität Marburg, 35032 Marburg, Germany

E-mail: frederikmichael.schiller@ehu.eus

\footnotetext{
${ }^{*}$ To whom correspondence should be addressed

${ }^{\dagger}$ Fachbereich Physik und Zentrum für Materialwissenschaften, Philipps-Universität Marburg, 35032 Marburg, Germany

$\ddagger$ Institut für Festkörperphysik, TU Dresden, D-01062 Dresden, Germany

ฯ Centro de Física de Materiales (CSIC-UPV-EHU) and Materials Physics Center (MPC), 20018 San Sebastián, Spain

$\S$ Donostia International Physics Center, 20018 Donostia-San Sebastián, Spain

"Catalan Institute of Nanoscience and Nanotechnology (ICN2), CSIC and The Barcelona Institute of Science and Technology, Campus UAB, Bellaterra, 08193 Barcelona, Spain

${ }^{\perp}$ ICREA, Institució Catalana de Recerca i Estudis Avançats, Lluis Companys 23, 08010 Barcelona, Spain

\#Fachbereich Chemie, Philipps-Universität Marburg, 35032 Marburg, Germany
} 


\begin{abstract}
We have investigated the electronic structures of axially oxo functionalized titanylphthalocyanine (TiOPc) on $\mathrm{Ag}(111)$ by X-ray and ultraviolet photoelectron spectroscopy, two-photon photoemission, X-ray absorption spectroscopy, and X-ray magnetic circular dichroism. Furthermore, we use complementary data of $\mathrm{TiOPc}$ on graphite and planar copper phthalocyanine $(\mathrm{CuPc})$ on $\mathrm{Ag}(111)$ for a comparative analysis. Both molecules adsorb on $\mathrm{Ag}(111)$ in a parallel orientation to the surface, for TiOPc with an oxygen-up configuration. The interaction of nitrogen and carbon atoms with the substrate is similar for both molecules while the bonding of the titanium atom to $\mathrm{Ag}(111)$ in the monolayer is found to be slightly more pronounced than in the $\mathrm{CuPc}$ case. Ultraviolet photoemission spectroscopy reveals an occupation of the lowest unoccupied molecular orbital (LUMO) level in monolayer thick TiOPc on $\mathrm{Ag}(111)$ related to the interaction of the molecules and the silver substrate. This molecule-metal interaction also causes an upward shift of the $\mathrm{Ag}(111)$ Shockley state that is transformed into an unoccupied interface state with energies of 0.23 and $0.33 \mathrm{eV}$ for the $\mathrm{TiOPc}$ mono- and bilayer, respectively, at the Brillouin zone center.
\end{abstract}

\title{
Introduction
}

The electronic properties of organic semiconductor interfaces on adequate substrates is of major importance for the development of devices based on molecular electronics. This is the case of organic photovoltaic cell (OPVCs) or organic light emitting diodes (OLEDs). The determination of the binding energies of the highest occupied molecular orbital (HOMO) and lowest unoccupied molecular orbital (LUMO) states of organic layers with respect to the Fermi level of the metal substrate is crucial, since these values define the energy barriers for hole and electron injection from the metal to the organic layer. Both, transparent insulating or semiconducting substrates but also metals have to be addressed here. ${ }^{1}$ The latter case presents the metal electrode contact of the organic semiconductor in the device. While 
the growth of these structures is often well known, ${ }^{2,3}$ the electronic structure has been less investigated. ${ }^{4}$ Here we analyze the electronic properties of titanylphthalocyanine (titanyl-Pc or TiOPc), molecules that are well known as an organic p-type semiconductor and photoconductor, ${ }^{5,6}$ that have been widely used in color laser printer toners but also have ability in further optoelectronic applications. ${ }^{7-9} \mathrm{TiOPc}$ is an oxometal phthalocyanine that has an axial $\mathrm{TiO}$ group with the oxygen atom protruding from the Pc molecular plane. As a result the molecule contains a strong dipole moment $P$ calculated to have values between 1.43 and $3.73 \mathrm{D},{ }^{10,11}$ which is absent in planar Pc molecules, e.g., copper phthalocyanine $(\mathrm{CuPc})$.

Several studies found that TiOPc on graphite (Highly Oriented Pyrolytic Graphite HOPG) grows with its backbone parallel to the surface plane and with the oxygen atom pointing away from the substrate. ${ }^{12-14}$ The same has been observed for other non-planar Pc molecules like VOPc ${ }^{15,16}$ or AlCl-Pc. ${ }^{17,18}$ However, some studies devoted to TiOPc on $\operatorname{Ag}(111)$ suggested a tilted orientation, ${ }^{19,20}$ that could not be confirmed by more recent investigations. ${ }^{21,22}$ These latest studies also suggest that the oxygen atom is pointing to the vacuum in the monolayer. Molecules in the second layer grow on top of the first one with the oxygen atom facing towards the surface in order to minimize the dipole-dipole interaction between layers ${ }^{23}$ (see Figure 1 for a sketch). The different layer morphologies reveal characteristic molecular arrangements on the surface of $\operatorname{Ag}(111)$ ranging from the submonolayer till the completion of the monolayer (ML) and the bilayer formation. Prior to the completion of a full TiOPc ML the molecules display a commensurate phase (c-phase) that develops into a point-on-line (POL) phase when the thickness is close to a full ML. The POL phase is characterized by a loss of the fixed 2D registry between molecules and the $\operatorname{Ag}(111)$ surface, while the unit cell vectors shift along the directions of the Ag atom rows. ${ }^{24}$ In this phase the molecules arrange in order to optimize their positions and azimuthal alignment within the layer. At full saturation (1ML) the short-range intermolecular repulsion becomes dominant, which causes an azimuthal reorientation in order to minimize repulsive overlap of neighboring species. ${ }^{21}$ The $1 \mathrm{ML}$ POL phase of $\mathrm{TiOPc}$ has a molecular area 
occupation of $199 \AA^{2}$, which corresponds to one molecule over nearly $28 \mathrm{Ag}$ atoms. ${ }^{21}$ This molecular density is slightly lower compared to $\mathrm{CuPc}^{25}$ that can compress more and occupy $192 \AA^{2}$. In both cases the molecules lie flat on the substrate and the small difference in the occupation is related to slight differences in both POL phases. In the case of TiOPc SPA-LEED analysis suggests a partially loss of registry with the $\operatorname{Ag}(111)$ surface, while in $\mathrm{CuPc}$ the loss of registry is even larger allowing a higher compression. The unit cell in both POL phases presents a squashed square with nearly identical base vectors and angles close to $90^{\circ} .{ }^{21,22,25}$ Therefore, on the hexagonal surface of the substrate, the molecules grow in three rotational domains. The formation of the second layer takes place when the ML is completely compressed and no further molecules can be incorporated in the first layer. ${ }^{23}$ All stages in that growth process display characteristic scanning tunneling microscopy (STM) and low energy electron diffraction (LEED) patterns that allow the identification of each phase depending on coverage. In this work we have used these characterization techniques for thickness calibration purposes prior to the electronic structure characterization of the layers.

Here we present a comprehensive analysis of the electronic structure of $\mathrm{TiOPc}$ grown on $\mathrm{Ag}(111)$ by means of X-ray, ultraviolet and two-photon photoemission spectroscopy as well as X-ray absorption and partially compare these results to the same molecule on a graphite (HOPG) surface, where the molecule-substrate interaction is reduced, and to flat $\mathrm{CuPc}$ molecules on $\mathrm{Ag}(111)$. We observe that the first TiOPc layer is weakly chemisorbed to $\operatorname{Ag}(111)$ confirming the oxygen-up orientation. On the other hand, already the second layer displays the same electronic properties as thick TiOPc films. The position of the characteristic HOMO level of the first monolayer adsorbed on $\mathrm{Ag}(111)$ still persists at the same energy due to the charge transfer between the molecular layer and the substrate independent of the additional layers evaporated on top of it. Additionally, a gradual energy shift of the HOMO level during the completion of the second layer is observed, which seems to be directly linked with the progressive change observed in the work function. At the same time the LUMO 
level that was found to be occupied in the monolayer range is vanishing with increasing thickness. From X-ray photoemission spectroscopy (XPS) data, we find that the strongest interaction of the molecules is by the nitrogen and carbon atoms. The axial Ti atom interaction with the substrate is small but not negligible. X-ray absorption spectra reveal a flat-lying configuration of the TiOPc molecules on $\mathrm{Ag}(111)$ excluding a tilt angle phase arrangement, a result that confirms recent investigations. ${ }^{21,22}$ This spectroscopy technique also confirms the partial occupation of the LUMO level in the monolayer case. X-ray magnetic circular dichroism experiments of the ML film reveal the absence of paramagnetism on the Ti atom. Furthermore, two-photon photoemission spectroscopy indicates the transformation of the $\operatorname{Ag}(111)$ Shockley state into an unoccupied interface state after absorption of TiOPc which shifts up in energy when going from a TiOPc mono- to a bilayer.

\section{Methods}

The photoemission (PE) experiments were performed at the Philipps-University of Marburg (Germany) while the X-ray absorption experiments were taken at the BOREAS beamline of ALBA synchrotron in Barcelona (Spain). Both experimental systems were equipped with Low Energy Electron Diffraction (LEED) to check for the ordering of the molecular layers. The $\operatorname{Ag}(111)$ crystal was prepared by repeated cycles of sputtering $(700 \mathrm{~V})$ and annealing $\left(500^{\circ} \mathrm{C}\right)$ until the $\operatorname{Ag}(111)$ surface state was well observed (Marburg) and the Scanning Tunneling Microscope indicated large flat terraces (Barcelona).

TiOPc was synthesized by the reaction of 1,2-dicyanobenzene and tetrabutoxy titanium modifying the established workup procedure. ${ }^{26}$ Purification was accomplished by extensive continuous hot-extraction of by-products by dichloroethane and acetonitrile $\left(80^{\circ} \mathrm{C}, 2 \mathrm{~h}\right.$ each). The remaining microcrystalline product was degassed at $400^{\circ} \mathrm{C}$ under vacuum conditions $\left(10^{-6} \mathrm{mbar}\right)$ for $4 \mathrm{~h}$ and analyzed by Electron Ionization Mass Spectroscopy (EI-MS), combustion analysis and Attenuated total reflection Infrared Spectroscopy (ATR-IR). The TiOPc 
molecules were then further purified in UHV by holding the evaporator temperature approx. $50 \mathrm{~K}$ below the deposition temperature for several weeks. This procedure ensured highly pure TiOPc molecules during deposition. Then TiOPc and $\mathrm{CuPc}$ (Aldrich 99\%) were deposited by a very small deposition rate (1-2 ML/hour) with the sample hold at $100^{\circ} \mathrm{C}$ in order to facilitate ordering of the layers. The base pressure of the system was $3 \times 10^{-11} \mathrm{mbar}$ and rised to $6 \times 10^{-11}$ mbar during deposition. The layer thickness was obtained by a combined analysis of the channelplate based Low Energy Electron Diffraction (OCI Vacuum Microeng. Inc.), evaporation time, and X-ray photoemission spectroscopy analysis of the attenuation of the substrate Ag $3 d$ core level emission in Marburg and by Scanning Tunneling Microscopy in Barcelona. XPS and UPS were obtained by Specs XR50 Al $K_{\alpha}$ emission and with a VG Microtech UVL-HI UPS/1 Helium lamp, respectively. The features due to the He I satellites were removed by numerical methods whose parameters were obtained for thick molecular films observing the $\mathrm{Ag}$ low density of states region between $4 \mathrm{eV}$ binding energy and the Fermi level. The work function and the two-photon photoemission data were measured with low energy photons produced independently from visible and ultraviolet photons delivered by an optical parametric oscillator (OPO) setup. X-ray absorption (XAS) data were taken as a total yield signal normalized to a gold mesh absorption inserted in the beamline. The light incidence angle was $20^{\circ}$ with respect to the surface for carbon and nitrogen $1 s$ absorption using horizontal and vertical polarized light. The measurement temperature for the normal XAS experiment was $300 \mathrm{~K}$. For the Ti $2 p$ measurement the sample was cooled to 3 $\mathrm{K}$ and rotated to normal incidence. In the last case for the X-ray magnetic circular dichroism experiment left and right circular polarized light was used and the magnetic field was applied perpendicular to the sample and along the incident photon propagation direction. STM images were taken both at Philipps-University of Marburg and at ALBA synchrotron of Barcelona using Omicron VT and Specs Aarhus instruments, respectively. 


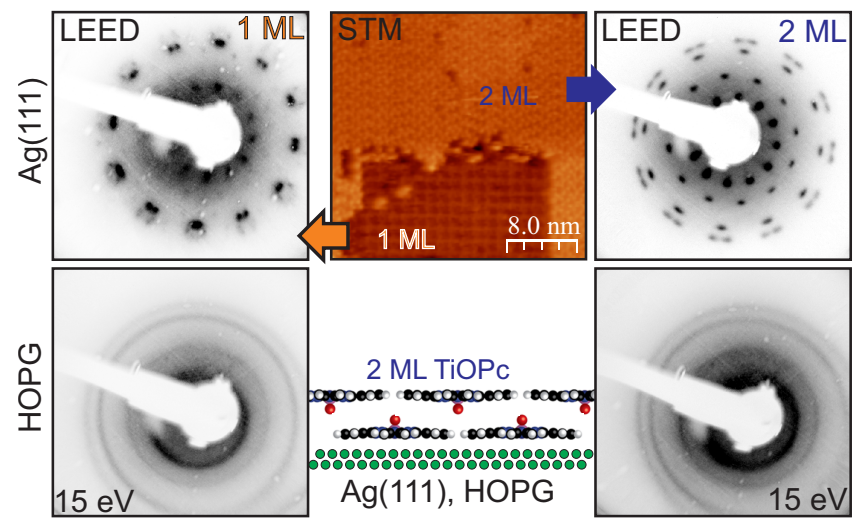

Figure 1: Growth of TiOPc on $\mathrm{Ag}(111)$ and HOPG, respectively. The monolayer and bilayer have characteristic real-spece images and patterns in the STM and LEED, respectively, that can be used to calibrate the TiOPc source. The HOPG substrate consists of small graphite flakes with a large number of rotational domains and a common perpendicular $c$-axis giving rise to the ring-like structures in the LEED.

\section{Results and Discussion}

\section{Structural analysis}

Figure 1 displays LEED patterns measured on $\mathrm{TiOPc} / \mathrm{Ag}(111)$, which display a POL phase measured for the compressed ML on TiOPc and a completely different LEED pattern for the TiOPc bilayer that is caused by the different molecular arrangement in the second layer. Additionally, the STM image shows a large area with bilayer formation together with a patch of the compressed ML. For comparison TiOPc layers were grown on a graphite (HOPG) surface, which is well known for a very weak interaction with the molecular layers. The HOPG substrate consists of micron-size crystallites having different rotational orientations but a common perpendicular axis. This arrangement give rise to ring like LEED patterns of the pure substrate. One, therefore, also expects ring-like diffraction structures of TiOPc molecules grown on this substrate. The ring diameters are very similar to the LEED spots of the same molecules on $\mathrm{Ag}(111)$, taking into account the rotational domains of the HOPG substrate and reveal only minor differences between 1 and 2 ML coverage. 


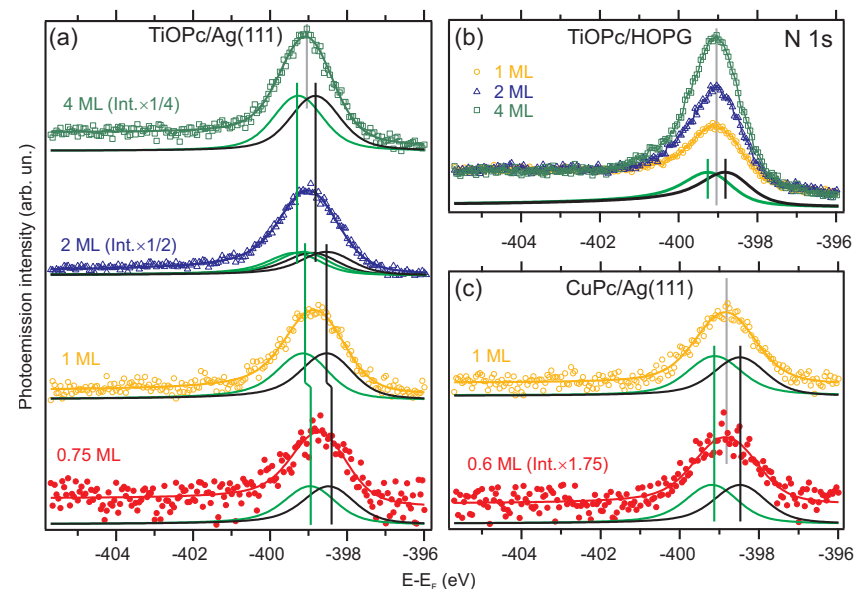

Figure 2: X-ray photoelectron spectroscopy taken at the N 1s level of TiOPc on $\mathrm{Ag}(111)$ (a) and HOPG (b) as well as $\mathrm{CuPc}$ on $\mathrm{Ag}(111)$ (c), respectively. The spectra were fitted by two individual but identical Doniach Sunjic peaks ${ }^{27}$ that are indicated below the data points [in (b) shown only for the monolayer thick film].

\section{Core level analysis}

XPS measurements were performed at the $\mathrm{C} 1 s, \mathrm{~N} 1 s$, and Ti $2 p$ regions for TiOPc layers with different thickness (0.75 ML - $4 \mathrm{ML}$ ) grown on $\mathrm{Ag}(111)$ and HOPG. For comparison similar measurements were carried out at the $\mathrm{N}$ and $\mathrm{C} 1 s$ and $\mathrm{Cu} 2 p$ regions on the $\mathrm{CuPc} / \mathrm{Ag}(111)$ system. Figures 2(a) and (b) compare the $\mathrm{N} 1 s$ emission for TiOPc layers grown with different thickness on $\mathrm{Ag}(111)$ and $\mathrm{HOPG}$, respectively, while in Figure 2(c) the CuPc results are shown. Molecule-substrate interaction between phthalocyanine molecules and HOPG is rather small, therefore, molecule-molecule interactions dominate in this system. ${ }^{13}$ On a more reactive metal surface like $\mathrm{TiOPc} / \mathrm{Ag}(111)$, the molecule-metal interaction is stronger, which is reflected in shifts of the molecular core levels in the mono- and submonolayer range. Therefore, we will first consider the weak interacting molecule adsorption on HOPG. In the TiOPc/HOPG system one observes a rather broad peak at $E_{B}=399 \mathrm{eV}$ that changes its intensity according to the thickness but does not present a core level shift. The broad peak is produced by the sum of two contributions of non-equivalent nitrogen atoms within the Pc ligand with the lower/higher binding energy peaks corresponding to the four nitrogen atoms in the iminic/pyrrolic position, respectively. The individual peaks cannot be resolved 
due to the linewidth of the peaks and to the resolution of the experimental system. In FePc the peak separation was theoretically calculated to be $0.3 \mathrm{eV} .{ }^{28}$ In order to observe both contributions, a peak fit analysis has been carried out here by using two individual Doniach Sunjic peaks ${ }^{27}$ of the same width, asymmetry and Shirley background, ${ }^{29}$ that resulted in a nitrogen peaks distance of approximately $(0.55 \pm 0.2) \mathrm{eV}$ for all considered cases. The fit results are included in Figure 2. For TiOPc on $\mathrm{Ag}(111)$ a change in the $\mathrm{N} 1 s$ binding energy position as a function of thickness is detected. The $4 \mathrm{ML} \mathrm{N} 1 s$ spectrum is found to be identical to the $\mathrm{TiOPc} / \mathrm{HOPG}$ case, demonstrating that this film seems to be electronically isolated from the Ag surface and can be considered as a thick film case. The monolayer spectrum is shifted by $0.2 \mathrm{eV}$ to lower binding energy, $E_{B}=398.8 \mathrm{eV}$. A further shift of $0.1 \mathrm{eV}$ is observed for $0.75 \mathrm{ML}$ TiOPc. The nitrogen emission peaks of $2 \mathrm{ML}$ TiOPc have an intermediate peak position between the one and four ML samples that is best fitted by a superposition of the doublet emissions from the latter two cases. It is also notable that the peak width in the mono- and submonolayer cases is enhanced with respect to the bilayer and 4 ML film. This situation can be caused either by (i) different molecular adsorption sites on the substrate or (ii) due to a lifting of the LUMO level degeneracy upon partial charging, in combination with core-hole screening effects. ${ }^{30}$ In $\mathrm{CuPc} / \mathrm{Ag}(111)$ a $\mathrm{N} 1 s$ core level shift from the submonolayer to the complete ML range is not observed, thicker films were not investigated. The N $1 s$ energy positions of the monolayer thick CuPc film are very similar to the TiOPc case.

Concerning the analysis of the $\mathrm{C} 1 s$ core level of TiOPc and $\mathrm{CuPc}$, three different contributions are found, as has been observed in other Pc molecules, see Figure 3(a) and (b). First, a contribution from the 24 carbon atoms of the benzene rings with a binding energy of $284.9 \mathrm{eV}$ in the $4 \mathrm{ML} \mathrm{TiOPc} / \mathrm{Ag}(111)$ case (the thickest film evaporated here); second, a contribution from the $8 \mathrm{C}$ atoms in the pyrrole structures with an $E_{B}=286.4 \mathrm{eV}$; and third a shake-up satellite at $288.2 \mathrm{eV}$. These $4 \mathrm{ML}$ TiOPc binding energy values are very similar to the ones of a $200 \mathrm{~nm} \mathrm{TiOPc} \mathrm{film.}{ }^{31}$ By lowering the thickness to $2 \mathrm{ML}$, a $0.1 \mathrm{eV}$ core level 


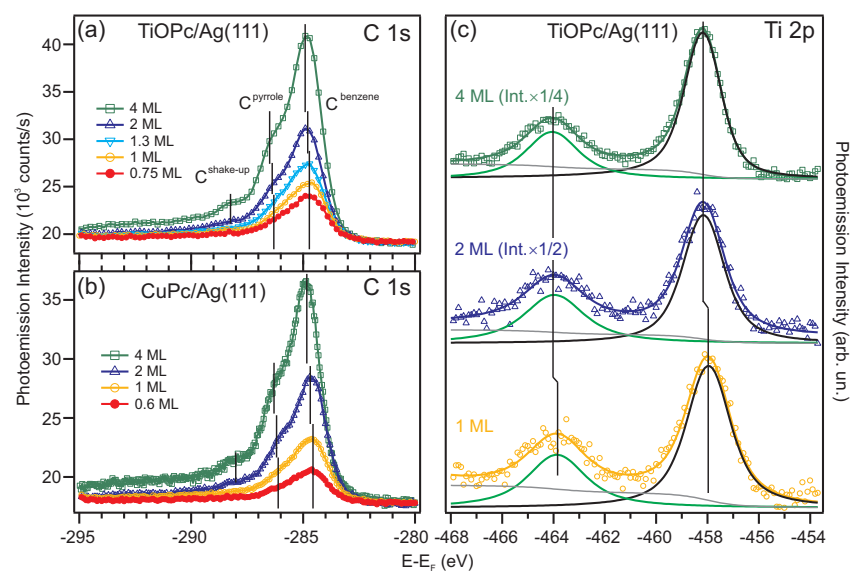

Figure 3: X-ray photoelectron spectroscopy taken at the $\mathrm{C} 1 s$ emissions of $\mathrm{TiOPc}(\mathrm{a})$ and $\mathrm{CuPc}(\mathrm{b})$ as well as at the $\mathrm{Ti} 2 p$ core levels (c) of TiOPc on $\mathrm{Ag}(111)$, respectively.

shift to lower binding energies is observed with respect to the thick layer case. For ML and sub-ML coverage, a further core level shift is observed, that amounts to $0.3 \mathrm{eV}$ with respect to the thick layer. In the case of $\mathrm{CuPc}$ the multi- to monolayer shift is identical to TiOPc films. The $\mathrm{C} 1 s$ core level analysis of the molecules on HOPG is complicated due to the overlapping of the substrate and molecular carbon atom emissions and was not carried out.

Figure 3(c) shows the $\mathrm{Ti} 2 p$ core level for $\mathrm{TiOPc} / \mathrm{Ag}(111)$. For comparison, in TiOPc/HOPG the Ti $2 p$ core level emission peaks were found independent of the film thickness at $E_{B}=$ 458.1 and $464.0 \mathrm{eV}$ for the $2 p_{3 / 2}$ and $2 p_{1 / 2}$ emissions, respectively (not shown). Identical energy positions as for TiOPc/HOPG were encountered for 2 and 4 ML TiOPc/Ag(111), while a core level shift of $(170 \pm 30) \mathrm{meV}$ to lower binding energies occurs for the $1 \mathrm{ML}$ case. For the weak interacting $\mathrm{CuPc} / \mathrm{Ag}(111)$ system, a $\mathrm{Cu} 2 p_{3 / 2}$ core level shift of less than $100 \mathrm{meV}$ from the submonolayer to the ML thickness has been observed (not shown). In this case the weak $\mathrm{Cu}-\mathrm{Ag}$ interaction is related to the fact that the $d_{x^{2}-y^{2}}$ levels near the Fermi energy are confined within the molecular plane. For strongly interacting central atoms of Pc complexes, like Fe or $\mathrm{Co}$ in FePc or $\mathrm{CoPc}$, core level shifts of more than $1 \mathrm{eV}$ have been found. ${ }^{32,33}$ In the last cases similar to iron or cobalt tetraphenylporphyrines (FeTPP, CoTPP) an Fe- or Co-Ag bond is formed that can be well described by the surface trans effect. ${ }^{34}$ In CoPc, as a result of such a strong interaction on a surface, the Co atom loses the gas-phase magnetic 

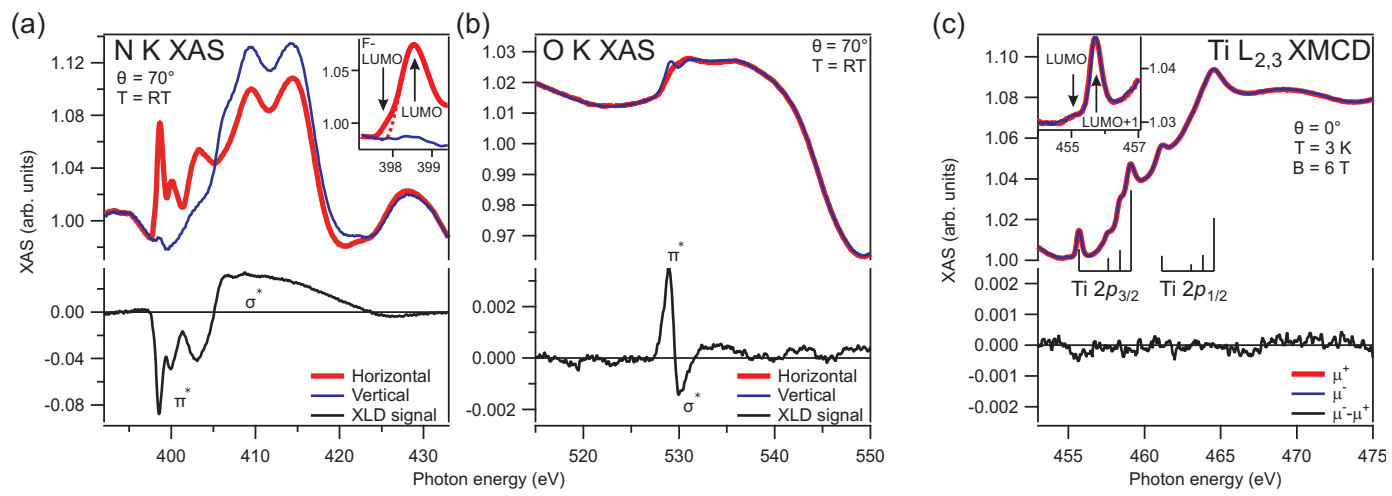

Figure 4: X-ray absorption spectroscopy measurements on $1 \mathrm{ML} \mathrm{TiOPc} / \mathrm{Ag}(111)$. (a), (b) $\mathrm{N}$ and $\mathrm{O}$ K-edge absorption for horizontal and vertical light polarization, respectively. The inset amplifies the region of the onset revealing a shoulder at the low energy side, the dashed line is a guide to the eye to observe the sholder. (c) $\mathrm{Ti} \mathrm{L}_{2,3}$ edge measured with circular polarized light at $T=3 \mathrm{~K}$ and $\mu_{0} H=6 \mathrm{~T}$. No paramagnetic signal is observed. Also here in the inset the onset region is amplified.

moment. ${ }^{35}$ We can compare the small core level shift for $\mathrm{Ti}$ in $\mathrm{TiOPc} / \mathrm{Ag}(111)$ also to the $\mathrm{V}$ $2 p$ core level shift in vanadyl-Pc (VOPc) on $\mathrm{Ag}(111)$ that was found to be $0.2 \mathrm{eV}$ between 1 and 10 ML thickness. ${ }^{36}$ On the contrary, VPc arguably the planar counterpart to VOPc, presents a core level shift of $0.9 \mathrm{eV}$ between the multilayer and monolayer film. ${ }^{37}$ The latter result signifies a much stronger interaction of the $\mathrm{V}$ atoms with $\mathrm{Ag}(111)$ than in the case of VOPc. In the $\mathrm{VOPc}$ case as well as for $\mathrm{TiOPc}_{\mathrm{O}}$ the strong $\mathrm{V}=\mathrm{O}$ and $\mathrm{Ti}=\mathrm{O}$ bond and a consequent small $\mathrm{V}-\mathrm{Ag}$ and $\mathrm{Ti}-\mathrm{Ag}$ interaction are considered responsible for the observed minor shifts.

\section{Lowest unoccupied molecular levels of TiOPc/Ag(111)}

The inner nitrogen-containing chromophore of the phthalocyanine ligand as well as carbon atoms will largely determine the frontier orbitals ${ }^{21}$ and, thereby, the optical absorption of the dye molecule. As a result the lowest energy nitrogen and carbon NEXAFS emissions are transitions into the LUMO levels while the titanium and oxygen transitions populate higher energy molecular levels, namely, the LUMO+1 level. ${ }^{21}$

Figures 4(a) and (b) show the NEXAFS absorption of the $\mathrm{N}$ and $\mathrm{O} K$-edges for linearly 
polarized light (using vertical and horizontal polarized light) at $\theta=70^{\circ}$ from the surface normal. The difference spectrum (X-ray linear dichroism, XLD) reveals information about the orientation of the molecule with respect to the surface. The $\mathrm{N} K$ edge measured with horizontal light polarization reveals a well defined peak structure around $400 \mathrm{eV}$, which is nearly absent with vertical polarization. The low energy emissions are due to the $\pi^{*}$ molecular orbitals while emissions above $405 \mathrm{eV}$ are related to the antibonding $\sigma^{*}$ transitions. The absence of the $\pi^{*}$ emissions in vertical polarization indicates that $1 \mathrm{ML}$ of TiOPc is adsorbed with the molecular backbone parallel to the $\mathrm{Ag}(111)$ surface, as is commonly observed in other ML thick phthalocyanines. ${ }^{38}$ This result confirms recent results concerning the planar adsorption geometry of $\mathrm{TiOPc}$ on $\mathrm{Ag}(111),{ }^{21}$ and is contrary to some works that suggest a partial tilt of TiOPc molecules adsorbed on $\mathrm{Ag}(111) .{ }^{20}$ The lowest two emission peaks are separated by $1.5 \mathrm{eV}$, again similar to the $1 \mathrm{ML} \mathrm{VOPc} / \mathrm{Ag}(111)$ case $^{36}$ and different from thick $\mathrm{TiOPc}^{31}$ or VOPc molecular multilayers. ${ }^{36,39}$ In the latter cases the energy distance is approximately $2 \mathrm{eV}$. Furthermore, if we focus on the onset [see inset in Figure 4(a)] we observe that the first peak has a shoulder on the low energy side, which is indicated by an arrow and by the dotted line below the peak. The peak separation from the leading peak is approx. $0.8 \mathrm{eV}$. For $\mathrm{F}_{16} \mathrm{CuPc}$ and $\mathrm{CuPc}$ on $\mathrm{Ag}(111)$, such a doublet was explained by the lifting of the LUMO degeneracy. ${ }^{30}$ Upon charge transfer from the substrate, the electronic energy can be minimized by splitting the two-fold degenerate $e_{g}$ LUMO into a partially filled F-LUMO and an empty LUMO levels. We ascribe our observation for $\mathrm{TiOPc} / \mathrm{Ag}(111)$ to the same effect.

The $\mathrm{O} 1 s$ absorption spectrum consists of a doublet peak at 529 and $530 \mathrm{eV}$ that is attributed to the transition from the $\mathrm{O} 1 s$ orbital to $\mathrm{Ti}=\mathrm{O} \pi^{*}$ and $\sigma^{*}$ orbitals, respectively. ${ }^{36}$ Both peaks reveal an angular dependence, and confirm that the $\mathrm{Ti}=\mathrm{O}$ bond is mainly oriented perpendicular to the surface. The main feature in the O K-edge XAS near $529 \mathrm{eV}$ is designated as a transition from the $\mathrm{O} 1 s$ core level to molecular LUMO+1 orbitals. The LUMO state in TiOPc/ $\operatorname{Ag}(111)$ mainly consists of $\mathrm{C}$ and $\mathrm{N} 2 p$ unoccupied orbitals, ${ }^{21}$ and 
there is no direct interaction between the $\mathrm{O}$ ion and the Pc ligand, similarly as in VOPc. ${ }^{36}$ Therefore, transitions to the LUMO levels are suppressed at the O K-edge. The O $1 s$ XLD curve resembles very well that observed for $1 \mathrm{ML} \operatorname{VOPc} / \operatorname{Ag}(111)$, which was assigned to molecules with oxygen-up orientation. In a hypothetical oxygen-down configuration like in $\operatorname{VOPc} / \mathrm{Si}(111)$, the $\pi^{*}$ peak would disappear due to the diminishing of the transition metaloxygen bond caused by surface atom-oxygen bond. ${ }^{36}$

Figure 4(c) shows the XAS at the Ti $2 p$ adsorption edge for circularly polarized light at normal incidence geometry and with a $6 \mathrm{~T}$ magnetic field applied perpendicular to the surface. The shape of the adsorption spectra was calculated in an early work of de Groot. ${ }^{40}$ The Ti $2 p_{3 / 2}$ adsorption edge of multilayer TiOPc reveals a multi-peak structure of 4 peaks that come from the lifting of degenerated $\mathrm{e}_{g}$ and $\mathrm{t}_{2 g}$ levels, while the Ti $2 p_{1 / 2}$ edge has less structural features due to lifetime broadenings. ${ }^{12,41}$ The Ti $2 p_{3 / 2}$ emission of the TiOPc ML shown here also consists of 4 peaks and is very similar to the more recent $\mathrm{TiOPc}$ publication ${ }^{41}$ from which the multiplet scheme used in the figure was taken. Similarly as observed in the case of oxygen, for Ti one expects transitions into the LUMO+1 level rather than the LUMO due to the very weak Ti contributions to the latter. ${ }^{21}$ As detailed in the inset in Figure 4(c), we observe a very faint pre-peak emission $0.7 \mathrm{eV}$ below the leading peak marked by an arrow in the inset that coincide well with the calculated LUMO - LUMO +1 distance. ${ }^{21}$ We assign this small feature to a weak transition into the LUMO level.

The $\mathrm{Ti}^{4+}$ ion in TiOPc has a $d^{0}$ electronic configuration that would lead to a diamagnetic behavior. ${ }^{42,43}$ In order to probe the magnetic properties, in the lower part of Figure 4(c) we show the X-ray magnetic circular dichroism signal taken at 3K and $6 \mathrm{~T}$ applied field. The same measurement on the $\mathrm{Cu}$ or $\mathrm{V} 2 p$ adsorption edge of $\mathrm{CuPc}^{44}$ or $\mathrm{VOPc}^{36}$ results in XMCD spectra that amount to approx. $20 \%$ of the total XAS peak height. Here in TiOPc the relative height of the XMCD signal is lower than 3\%. Therefore, we interpret this behavior as the absence of paramagnetism of the Ti atom as predicted earlier. In contrast, the $\mathrm{V}^{4+}$ ion in VOPc has a $d^{1}$ electronic configuration. The presence of an unpaired $3 \mathrm{~d}$ 


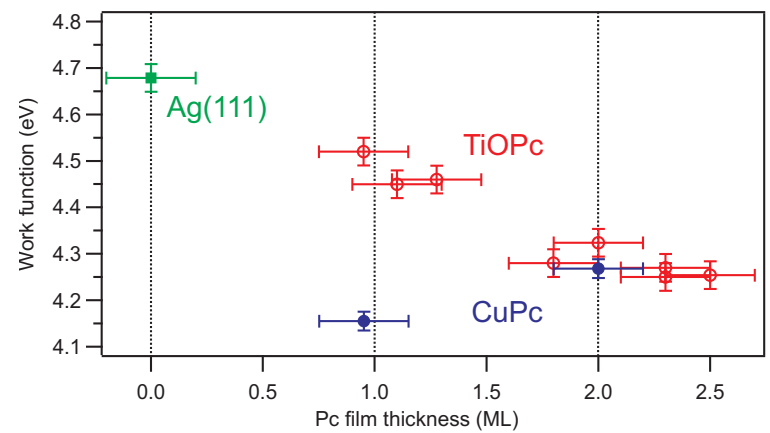

Figure 5: Work function measurements of $\mathrm{TiOPc}$ and $\mathrm{CuPc}$ layers as a function of thickness on $\mathrm{Ag}(111)$. While the $2 \mathrm{ML}$ molecular films have similar $\Phi$ values, due to the ordered $\mathrm{Ti}=\mathrm{O}$ dipoles in the TiOPc monolayer a much higher workfunction is observed.

electron results in a paramagnetic behavior that was also predicted earlier ${ }^{43}$ and observed by SQUID measurements for VOPc powder and by circular dichroism experiments for a monolayer thick VOPc film on $\operatorname{Ag}(111) .{ }^{36}$

\section{Work function and occupied band structure}

The work functions $\Phi$ were obtained from the secondary electron cutoff measurements of ultraviolet photoemission spectra where a small sample bias $(0.3$ to $0.5 \mathrm{~V})$ is applied in the normal emission geometry. For this purpose a frequency tripled laser pulse of a fundamental wavelength of 820 to $857 \mathrm{~nm}$ was focussed onto the sample. Figure 5 shows the result of the analysis. $2 \mathrm{ML}$ of $\mathrm{TiOPc}$ and $\mathrm{CuPc}$ results in a similar $\Phi=4.3 \mathrm{eV}$, while the ML values present strong deviations between the two molecules resulting in $\Phi^{\mathrm{CuPc}}=4.16 \mathrm{eV}$ and $\Phi^{\text {TiOPc }}=4.52 \mathrm{eV}$, respectively. The work function value measured here for $1 \mathrm{ML}$ of $\mathrm{CuPc}$ is identical to the reported one for $\mathrm{CuPc}^{45,46}$ and other planar $\mathrm{Pc}$ molecules grown on $\mathrm{Ag}(111)$, like $\mathrm{H}_{2} \mathrm{Pc}$ and CoPc. ${ }^{47}$ Also FePc reveals a work function values in the range $\Phi=4.15 \mathrm{eV}$ $4.3 \mathrm{eV} \cdot{ }^{45,48}$ In all these cases the strong Pauli push-back effect causes similar values of the work functions even if the bonding of the central metal atom differs in some cases. ${ }^{49}$ The much higher work function in the TiOPc monolayer results from the dipole-layer caused by the ordered axial $\mathrm{Ti}=\mathrm{O}$ groups with the oxygen atom pointing towards the vacuum. ${ }^{11,22}$ The value of the work function in $\mathrm{TiOPc} / \mathrm{Ag}(111)$ is, therefore, a combination of the dipole layer, 

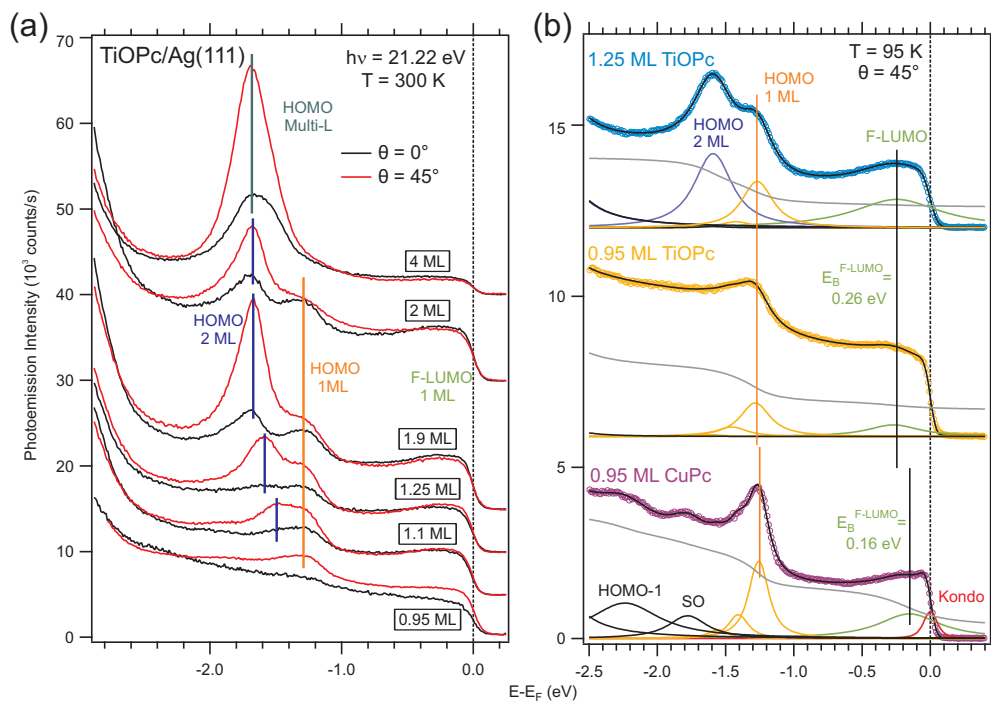

Figure 6: Ultraviolet photoemission spectroscopy of TiOPc molecules on $\mathrm{Ag}(111)$ as a function of thickness (a). The mono- and multi-layer emissions are marked. (b) Line fit analysis of $\mathrm{CuPc}$ as well as $\mathrm{TiOPc}$ molecular films close to the monolayer thickness.

the Pauli pushback effect and a depopulation of the $\operatorname{Ag}(111)$ surface state that occurs after molecule adsorption. ${ }^{22}$ The values observed here confirm qualitatively the observations from Kröger et al. ${ }^{22}$ nevertheless, we did not observe that the work function for 1 ML TiOPc has the same value as the work function of clean $\mathrm{Ag}(111)$. From the work function variation of $\mathrm{TiOPc}$ from 1 to $2 \mathrm{ML}$ and by taking into account that the dipolar layer contribution vanishes for the $2 \mathrm{ML}$ TiOPC system, we can calculate the dipolar moment of the TiOPc molecule. By assuming that the Pauli pushback effect and charge transfer effect do not vary from 1ML to 2ML and by using the Helmholtz classical electrostatic equation we get a value of $1.4 \mathrm{D}$ which is close to the value obtained by Fukagawa et al. ${ }^{11}$ by ultraviolet photoelectron spectroscopy measurements (1.77D) (see Supplementary Information for more detail).

The valence band emissions were measured by UV photoemission spectroscopy ( $h \nu=$ $21.2 \mathrm{eV}$ ) with the sample normal forming a $\theta=45^{\circ}$ angle to the analyzer. In this geometry the HOMO and F-LUMO emissions of most molecular features have been observed to have the highest cross section for the photon energy used here ${ }^{13,50-52}$ The evolution of the spectra as a function of TiOPc layer thickness is shown in Figure 6(a). The Fermi level position refers to that measured at the same geometry in the clean substrate previous to molecular 
adsorption. For comparison the $\theta=0^{\circ}$ spectra are also included. Similar to $\mathrm{CuPc}^{25}$ and other Pc molecules, the region near the Fermi level contains new emission peaks with respect to the clean $\operatorname{Ag}(111)$ substrate that are attributed to the molecules. The peak seen up to $2 \mathrm{ML}$ thickness at $1.25 \mathrm{eV}$ binding energy is the HOMO level of the first ML in contact with the $\operatorname{Ag}(111)$ surface. The emerging emission at higher binding energy is assigned to the HOMO of the $2 \mathrm{ML}$ film. It changes its energy position from $1.5 \mathrm{eV}$ to $1.7 \mathrm{eV}$ binding energy at the same time that the small 2 ML thick islands increase their area until the second layer is complete. This shift tracks the work function change of the system due to the vacuum level alignment of the physisorbed 2 ML HOMO level. ${ }^{22}$ For 4 ML TiOPc thickness, the important features related to the adsorption of the first layer, the HOMO and the F-LUMO level (see below), are no longer observed.

A peak fit analysis of the sub-ML and the $1.25 \mathrm{ML}$ TiOPc photoemission spectra is carried out and compared to a CuPc film of similar thickness, see Figure 6(b). The peak fit takes into account vibrational couplings of the HOMO levels ${ }^{53}$ that are present in $\mathrm{CuPc}^{54}$ and TiOPc/HOPG. ${ }^{10}$ Our analysis confirms these vibrational modes and energy positions already reported for $\mathrm{CuPc} / \mathrm{Ag}(111)^{25}$ and $\mathrm{TiOPc} / \mathrm{Ag}(111) .{ }^{22}$ There are additional features at higher binding energies of $1.8 \mathrm{eV}$ and $2.2 \mathrm{eV}$ in $\mathrm{CuPc}$ related to the split-off band (SO) of the HOMO- 1 and the HOMO-1 level itself, ${ }^{25}$ respectively. More importantly, there is an enhancement of the photoemission signal close to the Fermi level that derives from the LUMO levels of the molecules that appear as occupied states due to the strong charge transfer between $\mathrm{Ag}$ substrate and TiOPc molecules. This emission is marked as F-LUMO. Similar, relative broad F-LUMO peaks were observed in other molecular systems like PTCDA, ${ }^{55,56}$ $\mathrm{CuPc}^{25,30}$ or $\mathrm{SnPc}^{57}$ on $\mathrm{Ag}(111)$. For the peak-fitting process shown in Figure 6(b) we furthermore had to include a peak labeled as Kondo-peak at the Fermi energy position for a correct fitting. This last contribution has been observed previously on $\mathrm{CuPc} / \mathrm{Ag}(111)^{25}$ and derives from the many-body Kondo resonance that can arise from the interaction of localized spins, in this case that of the unpaired F-LUMO electron, with the surrounding electron sea 
of the metallic substrate. ${ }^{25,58}$ In TiOPc such a peak was not necessary to include. From the fit we observe that the F-LUMO level measured in $\mathrm{CuPc} / \mathrm{Ag}(111)$ coincide with previous $\operatorname{results}^{25,58}$ and is found at $0.16 \mathrm{eV}$ binding energy. In $\mathrm{TiOPc} / \mathrm{Ag}(111)$ the peak shifts to slightly higher binding energy at $0.26 \mathrm{eV}$. Due to the large linewidth of the LUMO emission peak $(500 \mathrm{meV})$, the error bar is in the same range as the peak shift. Nevertheless, also X-ray standing wave experiments revealed a smaller TiOPc molecular backbone-Ag distance than in $\mathrm{CuPc} / \mathrm{Ag}(111)$. This means that the $-i$ interaction between $\mathrm{TiOPc}_{\mathrm{O}}$ and $\mathrm{Ag}(111)$ is stronger than that between $\mathrm{CuPc}$ and $\mathrm{Ag}(111)$ and explains the higher binding energy in TiOPc.

\section{TiOPc-Ag interface state}

Time- and angle-resolved two-photon photoemission experiments on PTCDA/Ag(111) found an unoccupied interface state and concluded from the dispersion and the rather short inelastic lifetime of that state, slightly above $E_{F}$, that it must originate from the Shockley state of the bare $\operatorname{Ag}(111)$ substrate, which is upshifted from below the metallic Fermi level by as much as $0.7 \mathrm{eV}$ due to the interaction with the molecular layer. ${ }^{59,60}$ This interpretation was subsequently confirmed by density-functional theory (DFT) calculations. ${ }^{61-66}$ In the meantime, additional molecules, e.g., NTCDA, ${ }^{63} \mathrm{H}_{2} \mathrm{Pc}$ and $\mathrm{FePc}^{48}$ grown on $\mathrm{Ag}(111)$ and

other substrates have been investigated, ${ }^{67-79}$ which confirm the rather general phenomenon of the existence of interface states. Such a state can be successfully described by a model potential combining the metal substrate and a flat $\pi$-conjugated molecular layer. ${ }^{80}$

In order to check for a possible interface state (IS) close to the Fermi level $E_{F}$, we carried out two-photon photoemission (2PPE) experiments, since conventional one-photon photoemission did not show evidences for such a state (see normal emission spectra in Figure 6.) Figure 7(a) shows angle-resolved 2PPE data taken for 1.1 ML TiOPc. The UV photon energy was chosen right below the work function value to exclude one-photon photoemission processes. Here the visible-light photon pumps electrons into the unoccupied interface state 

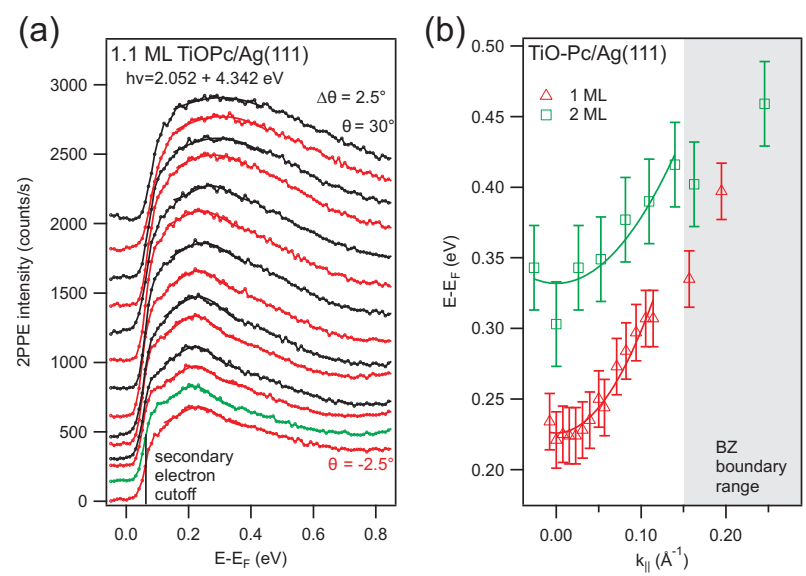

Figure 7: Two-photon photoemission spectroscopy of TiOPc molecules on $\mathrm{Ag}(111)$. (a): Interface state spectra as a function of the emission angle. (b) Energy positions and their fitted dispersion relations for different $\mathrm{TiOPc}$ molecular layer thickness. The grey area denotes the $k$ area close to the Brillouin zone boundary that was excluded from the fitting.

and the UV photon excites these electrons then above the vacuum level to be detected in the electron analyzer. In order to allow the detection of such state due to the reduced analyzer transmission at small kinetic energies of the emitted electrons, a voltage of $0.3 \mathrm{~V}$ has been applied to the sample. The Fermi level position is calculated from the measured value of the work function and crosschecked by the Shockley state energy position excited by one- and two-photon photoelectron emissions from the clean $\operatorname{Ag}(111)$ substrate. In the spectra right above the Fermi level, one observes the onset of 2PPE secondary electrons that are cutoff due to the reduced work function of the molecular layer followed by the the peak of the interface state. The position of this state changes with the emission angle $\theta$. From the emission angle the momentum values can be calculated by the relation $\hbar k_{\|}=\sqrt{2 m_{e} / E_{\text {kin }}} \sin \theta$ being $m_{e}$ the electron mass and $E_{\text {kin }}=h \nu-\Phi+E_{B+}$ the kinetic energy, where $E_{B+}$ represents the energy of the interface state with respect to the Fermi level. The dispersion of the interface states of TiOPc layers with different thickness is represented in Figure 7(b). The fittings of the dispersion relations by a parabolic function centered at $k_{\|}=0$ is included by solid lines, extracting the corresponding $E_{B+}$ values of $(0.23 \pm 0.02) \mathrm{eV}$ for the TiOPc ML and $(0.33 \pm 0.03)$ for the bilayer (spectra not shown). The fitting of the $k_{\|}$range was restricted to $k<0.15 \AA^{-1}$ to exclude deviations due to the Brillouin zone boundary of the molecular 
layer. For comparison, PTCDA ${ }^{59,81}$ and NTCDA ${ }^{63}$ interface states on $\mathrm{Ag}(111)$ have been found at $E_{B+}=0.6 \mathrm{eV}$ and $0.4 \mathrm{eV}$, respectively. These last molecules are strongly bound to the substrate with the consequence of a smaller molecule-metal distance and an upward shift of the state. ${ }^{80}$ For phthalocyanine molecules on $\mathrm{Ag}(111)$ only few data are available due to the small number of two-photon photoemission stations that allow the determination of the state energy in the unoccupied band structure. The IS positions have been determined for $\mathrm{H}_{2} \mathrm{Pc}$ and $\mathrm{FePc}^{48}$ at $E_{B+}=0.23$ and $0.31 \mathrm{eV}$, respectively. For FePc the upward shift has been explained by the strong Fe-Ag bonding causing a lower molecule-metal bonding and a stronger Fe $d$ orbital-based interaction. We also investigated the $\mathrm{CuPc}$ monolayer, but in this case due to its small work function $(\Phi=4.16 \mathrm{eV})$ and the limitation of the laser setup $(h \nu>4.3 \mathrm{eV})$, we were unable to obtain clear indications of the interface state. The effective mass of the $1 \mathrm{ML}$ TiOPc interface state was determined as $m_{\mathrm{eff}}=(0.53 \pm 0.12) \times m_{e}$, and for the $2 \mathrm{ML}$ film $m_{\mathrm{eff}}=(0.82 \pm 0.24) \times m_{e}$. The ML value is very similar to the ones encountered in $\mathrm{H}_{2} \mathrm{Pc}\left(m_{\mathrm{eff}}=0.5 \times m_{e}\right)$ or $\mathrm{FePc}\left(m_{\mathrm{eff}}=0.7 \times m_{e}\right)$. It seems that in general the values of the effective masses are slightly higher than the effective mass of the $\mathrm{Ag}(111)$ Shockley state $\left(m_{\mathrm{eff}}=0.4 \times m_{e}\right)^{82}$ and grow with increasing state energy in this systems. This points to an electronic corrugation of the interface state caused by the phthalocyanine molecules. The interface state is sensitive to the first angstroms near the metal-organic interface. ${ }^{83}$ Consequently, a decrease in molecule-metal distance, which is indicated by the increasing state energy, could enhance the corrugation of the interface state, leading to a higher effective mass. The polar titanyl group of TiOPc might act as an additional distancedependent source of corrugation and, indeed, even if the state energies are taken into account the effective masses measured for TiOPc seems to be slightly higher than those observed for $\mathrm{H}_{2} \mathrm{Pc}$ or FePc. Nevertheless, the experimental uncertainties limit a closer comparison. 


\section{Conclusions}

The electronic structure of non-planar TiOPc molecular layers on $\mathrm{Ag}(111)$ have been investigated and compared to the same material grown on HOPG and to planar CuPc molecules on $\mathrm{Ag}(111)$. From core-level photoemission and X-ray absorption experiments we confirm recent investigations that $\mathrm{TiOPc}$ molecules grow flat on $\mathrm{Ag}(111)$ with the main interaction of the nitrogen atoms with the silver and a rather weak interaction of the $\mathrm{Ti}$ atom. In this sense TiOPc behaves similar to VOPc, with one difference: the $d^{1} \mathrm{~V}^{4+}$ ion reveals a paramagnetic response to magnetic fields, whereas diamagnetic $d^{0} \mathrm{Ti}^{4+}$ does not show this response in $\mathrm{TiOPc} / \mathrm{Ag}(111)$. The molecular LUMO level of the monolayers was found to be partially occupied due to the charge transfer with the metallic substrate, the second layer is already decoupled from the substrate and the LUMO level is not longer occupied. Similar to other molecule/ $\operatorname{Ag}(111)$ systems the Shockley state shifts above the Fermi level and forms an unoccupied interface state. Its energy and dispersion could be analyzed by two-photon photoemission with an energy position of 0.23 and $0.33 \mathrm{eV}$ above the Fermi level for the mono- and bilayer, respectively.

\section{Supporting Information Available}

The Supporting Information contains information on the determination of the value of the dipole moment of $1 \mathrm{ML} \mathrm{TiOPc}$. This material is available free of charge via the Internet at http://pubs.acs.org/.

\section{Acknowledgement}

The authors acknowledge financial support from the Deutsche Forschungsgemeinschaft through SFB 1083 "Structure and Dynamics of Internal Interfaces", the Spanish CSIC I-Link programm, the Spanish MINECO (Grants No. MAT2016-78293-C6-2-R), the Severo Ochoa Centers of Excellence Program under Grant No. SEV-2013-0295. M. Paradinas thanks the 
Spanish Government for financial support through PTA2014-09788-I fellowships. ICN2 is funded by the CERCA Programme / Generalitat de Catalunya. We additionally acknowledge BOREAS beamline staff, especially Hari Babu, and ALBA for provision of synchrotron radiation and for synchrotron access funding.

\section{References}

(1) Ogawa, S., Ed. Organic Electronics Materials and Devices; Springer: Tokyo, 2015.

(2) Koch, N., Ueno, N., Wee, A. T. S., Eds. The Molecule-Metal Interface; Wiley-VCH Verlag GmbH \& Co. KGaA: Weinheim, 2013.

(3) Gottfried, J. M. Surface chemistry of porphyrins and phthalocyanines. Surface Science Reports 2015, 70, $259-379$.

(4) Mugarza, A.; Robles, R.; Krull, C.; Korytár, R.; Lorente, N.; Gambardella, P. Electronic and magnetic properties of molecule-metal interfaces: Transition-metal phthalocyanines adsorbed on $\operatorname{Ag}(100)$. Phys. Rev. B 2012, 85, 155437.

(5) Zhang, Z.; Jiang, L.; Cheng, C.; Zhen, Y.; Zhao, G.; Geng, H.; Yi, Y.; Li, L.; Dong, H.; Shuai, Z. et al. The Impact of Interlayer Electronic Coupling on Charge Transport in Organic Semiconductors: A Case Study on Titanylphthalocyanine Single Crystals. Angewandte Chemie International Edition 2016, 55, 5206-5209.

(6) Coppedè, N.; Bonnini, E.; Mezzadri, F.; Tarabella, G.; Ranzieri, P.; Barba, L.; Arrighetti, G.; Lutterotti, L.; Iannotta, S. Structural and morphological phase control by supersonic beams on titanyl phthalocyanine: An investigation on the growth. Organic Electronics 2016, 32, $15-20$.

(7) Mizuguchi, J.; Rihs, G.; Karfunkel, H. R. Solid-State Spectra of Titanylphthalocyanine As Viewed from Molecular Distortion. The Journal of Physical Chemistry 1995, 99, $16217-16227$. 
(8) Vasseur, K.; Rand, B. P.; Cheyns, D.; Temst, K.; Froyen, L.; Heremans, P. Correlating the Polymorphism of Titanyl Phthalocyanine Thin Films with Solar Cell Performance. The Journal of Physical Chemistry Letters 2012, 3, 2395-2400.

(9) Mayukh, M.; Macech, M. R.; Placencia, D.; Cao, Y.; Armstrong, N. R.; McGrath, D. V. Solution Processed Titanyl Phthalocyanines as Donors in Solar Cells: Photoresponse to $1000 \mathrm{~nm}$. ACS Applied Materials \&3 Interfaces 2015, 7, 23912-23919.

(10) Yamane, H.; Honda, H.; Fukagawa, H.; Ohyama, M.; Hinuma, Y.; Kera, S.; Okudaira, K.; Ueno, N. HOMO-band fine structure of OTi- and Pb-phthalocyanine ultrathin films: effects of the electric dipole layer. Journal of Electron Spectroscopy and Related Phenomena 2004, 137 - 140, 223 - 227.

(11) Fukagawa, H.; Yamane, H.; Kera, S.; Okudaira, K. K.; Ueno, N. Experimental estimation of the electric dipole moment and polarizability of titanyl phthalocyanine using ultraviolet photoelectron spectroscopy. Phys. Rev. B 2006, 73, 041302.

(12) Alfredsson, Y.; Rensmo, H.; Sandell, A.; Siegbahn, H. Electronic structure of thin film TiOPc studied by means of X-ray absorption and photoelectron spectroscopies. Journal of Electron Spectroscopy and Related Phenomena 2009, 174, 50 - 54.

(13) Kera, S.; Yamane, H.; Fukagawa, H.; Hanatani, T.; Okudaira, K.; Seki, K.; Ueno, N. Angle resolved $\{\mathrm{UV}\}$ photoelectron spectra of titanyl phthalocynine monolayer film on graphite. Journal of Electron Spectroscopy and Related Phenomena 2007, 156 - 158, $135-138$.

(14) Park, J. H.; Ravavar, L.; Kwak, I.; Fullerton-Shirey, S. K.; Choudhury, P.; Kummel, A. C. Growth Mode Transition from Monolayer by Monolayer to Bilayer by Bilayer in Molecularly Flat Titanyl Phthalocyanine Film. The Journal of Physical Chemistry C 2017, 121, 6721-6728. 
(15) Niu, T.; Zhou, C.; Zhang, J.; Zhong, S.; Cheng, H.; Chen, W. Substrate Reconstruction Mediated Unidirectionally Aligned Molecular Dipole Dot Arrays. The Journal of Physical Chemistry C 2012, 116, 11565-11569.

(16) Zhang, J.; Wang, Z.; Niu, T.; Li, Z.; Chen, W. Single molecule tunneling spectroscopy investigation of reversibly switched dipolar vanadyl phthalocyanine on graphite. Applied Physics Letters 2014, 104.

(17) Kera, S.; Yamane, H.; Honda, H.; Fukagawa, H.; Okudaira, K.; Ueno, N. Photoelectron fine structures of uppermost valence band for well-characterized ClAl-phthalocyanine ultrathin film: $\{$ UPS $\}$ and $\{$ MAES $\}$ study. Surface Science 2004, 566 - 568, Part 1, $571-578$.

(18) Fukagawa, H.; Hosoumi, S.; Yamane, H.; Kera, S.; Ueno, N. Dielectric properties of polar-phthalocyanine monolayer systems with repulsive dipole interaction. Phys. Rev. B 2011, 83, 085304 .

(19) Wei, Y.; Robey, S. W.; Reutt-Robey, J. E. Flux-Selected Titanyl Phthalocyanine Monolayer Architecture on $\mathrm{Ag}$ (111). The Journal of Physical Chemistry C 2008, 112, 1853718542 .

(20) Liu, X.; Wei, Y.; Reutt-Robey, J. E.; Robey, S. W. Dipole-Dipole Interactions in TiOPc Adlayers on Ag. The Journal of Physical Chemistry C 2014, 118, 3523-3532.

(21) Fernández, L.; Thussing, S.; Mänz, A.; Witte, G.; Brion-Rios, A. X.; CabreraSanfelix, P.; Sanchez-Portal, D.; Jakob, P. Structural and Vibrational Properties of the TiOPc Monolayer on $\mathrm{Ag}(111)$. The Journal of Physical Chemistry C 2017, 121, $1608-1617$.

(22) Kröger, I.; Stadtmüller, B.; Kumpf, C. Submonolayer and multilayer growth of titaniumoxide-phthalocyanine on $\operatorname{Ag}(111)$. New Journal of Physics 2016, 18, 111002. 
(23) Fernandez, L.; Thussing, S.; Mänz, A.; Sundermeyer, J.; Witte, G.; Jakob, P. The discrete nature of inhomogeneity: the initial stages and local configurations of TiOPc during bilayer growth on $\mathrm{Ag}(111)$. Phys. Chem. Chem. Phys. 2017, 19, 2495-2502.

(24) Hooks, D. E.; Fritz, T.; Ward, M. D. Epitaxy and Molecular Organization on Solid Substrates. Advanced Materials 2001, 13, 227-241.

(25) Kröger, I.; Stadtmüller, B.; Stadler, C.; Ziroff, J.; Kochler, M.; Stahl, A.; Pollinger, F.; Lee, T.-L.; Zegenhagen, J.; Reinert, F. et al. Submonolayer growth of copperphthalocyanine on $\operatorname{Ag}(111)$. New Journal of Physics 2010, 12, 083038.

(26) Jiachang, Y.; Hisatomo, Y.; Chyongjin, P. A Convenient Synthetic Method for Pure Oxo(phthalocyaninato)titanium(IV) and Application to Other Metal Phthalocyanines. Bulletin of the Chemical Society of Japan 1995, 68, 1001-1005.

(27) Doniach, S.; Sunjic, M. Many-electron singularity in X-ray photoemission and X-ray line spectra from metals. Journal of Physics C: Solid State Physics 1970, 3, 285.

(28) Åhlund, J.; Nilson, K.; Schiessling, J.; Kjeldgaard, L.; Berner, S.; Mårtensson, N.; Puglia, C.; Brena, B.; Nyberg, M.; Luo, Y. The electronic structure of iron phthalocyanine probed by photoelectron and x-ray absorption spectroscopies and density functional theory calculations. The Journal of Chemical Physics 2006, 125, 034709.

(29) Shirley, D. A. High-Resolution X-Ray Photoemission Spectrum of the Valence Bands of Gold. Phys. Rev. B 1972, 5, 4709-4714.

(30) Borghetti, P.; El-Sayed, A.; Goiri, E.; Rogero, C.; Lobo-Checa, J.; Floreano, L.; Ortega, J. E.; de Oteyza, D. G. Spectroscopic Fingerprints of Work-Function-Controlled Phthalocyanine Charging on Metal Surfaces. ACS Nano 2014, 8, 12786-12795, PMID: 25426520. 
(31) Zhang, Y.; Wang, S.; Demasi, A.; Reid, I.; Piper, L. F. J.; Matsuura, A. Y.; Downes, J. E.; Smith., K. E. Soft X-ray spectroscopy study of electronic structure in the organic semiconductor titanyl phthalocyanine (TiO-Pc). J. Mater. Chem. 2008, $18,1792-1798$.

(32) Petraki, F.; Peisert, H.; Aygül, U.; Latteyer, F.; Uihlein, J.; Vollmer, A.; Chassé, T. Electronic Structure of FePc and Interface Properties on $\mathrm{Ag}(111)$ and $\mathrm{Au}(100)$. The Journal of Physical Chemistry C 2012, 116, 11110-11116.

(33) Petraki, F.; Peisert, H.; Biswas, I.; Chassé, T. Electronic Structure of CoPhthalocyanine on Gold Investigated by Photoexcited Electron Spectroscopies: Indication of Co IonMetal Interaction. The Journal of Physical Chemistry C 2010, 114, $17638-17643$.

(34) Hieringer, W.; Flechtner, K.; Kretschmann, A.; Seufert, K.; Auwärter, W.; Barth, J. V.; Görling, A.; Steinrück, H.-P.; Gottfried, J. M. The Surface Trans Effect: Influence of Axial Ligands on the Surface Chemical Bonds of Adsorbed Metalloporphyrins. Journal of the American Chemical Society 2011, 133, 6206-6222.

(35) Stepanow, S.; Miedema, P. S.; Mugarza, A.; Ceballos, G.; Moras, P.; Cezar, J. C.; Carbone, C.; de Groot, F. M. F.; Gambardella, P. Mixed-valence behavior and strong correlation effects of metal phthalocyanines adsorbed on metals. Phys. Rev. B 2011, 83, 220401.

(36) Eguchi, K.; Takagi, Y.; Nakagawa, T.; Yokoyama, T. Molecular Orientation and Electronic States of Vanadyl Phthalocyanine on $\mathrm{Si}(111)$ and $\mathrm{Ag}(111)$ Surfaces. The Journal of Physical Chemistry C 2013, 117, 22843-22851.

(37) Eguchi, K.; Nakagawa, T.; Takagi, Y.; Yokoyama, T. Direct Synthesis of Vanadium Phthalocyanine and Its Electronic and Magnetic States in Monolayers and Multilayers on $\mathrm{Ag}(111)$. The Journal of Physical Chemistry C 2015, 119, 9805-9815. 
(38) Rocco, M. L. M.; Frank, K.-H.; Yannoulis, P.; Koch, E.-E. Unoccupied electronic structure of phthalocyanine films. The Journal of Chemical Physics 1990, 93, 6859-6864.

(39) Zhang, Y.; Learmonth, T.; Wang, S.; Matsuura, A. Y.; Downes, J.; Plucinski, L.; Bernardis, S.; O’Donnell, C.; Smith, K. E. Electronic structure of the organic semiconductor vanadyl phthalocyanine (VO-Pc). J. Mater. Chem. 2007, 17, 1276-1283.

(40) de Groot, F. X-ray absorption and dichroism of transition metals and their compounds. Journal of Electron Spectroscopy and Related Phenomena 1994, 67, 529 - 622.

(41) Pickup, D. F.; Zegkinoglou, I.; Ballesteros, B.; Ganivet, C. R.; García-Lastra, J. M.; Cook, P. L.; Johnson, P. S.; Rogero, C.; de Groot, F.; Rubio, A. et al. Influence of Axial and Peripheral Ligands on the Electronic Structure of Titanium Phthalocyanines. The Journal of Physical Chemistry C 2013, 117, 4410-4420.

(42) Taube, R. Über Titanphthalocyanine. Zeitschrift für Chemie 1963, 3, 194-194.

(43) Lever, A. B. P. 336. The magnetic behaviour of transition-metal phathalocyanines. J. Chem. Soc. 1965, 1821-1829.

(44) Stepanow, S.; Mugarza, A.; Ceballos, G.; Moras, P.; Cezar, J. C.; Carbone, C.; Gambardella, P. Giant spin and orbital moment anisotropies of a $\mathrm{Cu}$-phthalocyanine monolayer. Phys. Rev. B 2010, 82, 014405.

(45) Muller, E. A.; Johns, J. E.; Caplins, B. W.; Harris, C. B. Quantum confinement and anisotropy in thin-film molecular semiconductors. Phys. Rev. B 2011, 83, 165422.

(46) Huang, Y. L.; Wruss, E.; Egger, D. A.; Kera, S.; Ueno, N.; Saidi, W. A.; Bucko, T.; Wee, A. T.; Zojer, E. Understanding the Adsorption of $\mathrm{CuPc}$ and $\mathrm{ZnPc}$ on Noble Metal Surfaces by Combining Quantum-Mechanical Modelling and Photoelectron Spectroscopy. Molecules 2014, 19, 2969. 
(47) Caplins, B. W.; Shearer, A. J.; Suich, D. E.; Muller, E. A.; Harris, C. B. Measuring the electronic corrugation at the metal/organic interface. Phys. Rev. B 2014, 89, 155422.

(48) Caplins, B. W.; Suich, D. E.; Shearer, A. J.; Harris, C. B. Metal/Phthalocyanine Hybrid Interface States on $\operatorname{Ag}(111)$. The Journal of Physical Chemistry Letters 2014, 5, 16791684.

(49) Witte, G.; Lukas, S.; Bagus, P. S.; Wöll, C. Vacuum level alignment at organic/metal junctions: "Cushion" effect and the interface dipole. Applied Physics Letters 2005, 87.

(50) Hasegawa, S.; Tanaka, S.; Yamashita, Y.; Inokuchi, H.; Fujimoto, H.; Kamiya, K.; Seki, K.; Ueno, N. Molecular orientation in thin films of bis(1,2,5-thiadiazolo)- $p$ quinobis(1,3-dithiole) on graphite studied by angle-resolved photoelectron spectroscopy. Phys. Rev. B 1993, 48, 2596-2600.

(51) Kera, S.; Tanaka, S.; Yamane, H.; Yoshimura, D.; Okudaira, K.; Seki, K.; Ueno, N. Quantitative analysis of photoelectron angular distribution of single-domain organic monolayer film: $\{$ NTCDA $\}$ on GeS(0 0 1). Chemical Physics 2006, 325, 113 - 120 .

(52) Ziroff, J.; Forster, F.; Schöll, A.; Puschnig, P.; Reinert, F. Hybridization of Organic Molecular Orbitals with Substrate States at Interfaces: PTCDA on Silver. Phys. Rev. Lett. 2010, 104, 233004.

(53) Ueno, N.; Kera, S.; Sakamoto, K.; Okudaira, K. K. Energy band and electron-vibration coupling in organic thin films: photoelectron spectroscopy as a powerful tool for studying the charge transport. Applied Physics A 2008, 92, 495-504.

(54) Kera, S.; Yamane, H.; Sakuragi, I.; Okudaira, K. K.; Ueno, N. Very narrow photoemission bandwidth of the highest occupied state in a copper-phthalocyanine monolayer. Chemical Physics Letters 2002, 364, 93 - 98. 
(55) Kraft, A.; Temirov, R.; Henze, S. K. M.; Soubatch, S.; Rohlfing, M.; Tautz, F. S. Lateral adsorption geometry and site-specific electronic structure of a large organic chemisorbate on a metal surface. Phys. Rev. B 2006, 74, 041402.

(56) Puschnig, P.; Reinisch, E.-M.; Ules, T.; Koller, G.; Soubatch, S.; Ostler, M.; Romaner, L.; Tautz, F. S.; Ambrosch-Draxl, C.; Ramsey, M. G. Orbital tomography: Deconvoluting photoemission spectra of organic molecules. Phys. Rev. B 2011, 84 , 235427.

(57) Häming, M.; Scheuermann, C.; Schöll, A.; Reinert, F.; Umbach, E. Coverage dependent organic-metal interaction studied by high-resolution core level spectroscopy: $\mathrm{SnPc}$ (sub)monolayers on $\operatorname{Ag}\left(\begin{array}{lll}1 & 1 & 1\end{array}\right)$. Journal of Electron Spectroscopy and Related Phenomena 2009, 174, $59-64$.

(58) Ziroff, J.; Hame, S.; Kochler, M.; Bendounan, A.; Schöll, A.; Reinert, F. Low-energy scale excitations in the spectral function of organic monolayer systems. Phys. Rev. B 2012, 85, 161404.

(59) Schwalb, C. H.; Sachs, S.; Marks, M.; Schöll, A.; Reinert, F.; Umbach, E.; Höfer, U. Electron Lifetime in a Shockley-Type Metal-Organic Interface State. Phys. Rev. Lett. 2008, 101, 146801.

(60) Sachs, S.; Schwalb, C. H.; Marks, M.; Schöll, A.; Reinert, F.; Umbach, E.; Höfer, U. Electronic structure at the perylene-tetracarboxylic acid dianhydride/ $\operatorname{Ag}(111)$ interface studied with two-photon photoelectron spectroscopy. J. Chem. Phys. 2009, 131, 144701.

(61) Dyer, M. S.; Persson, M. The Nature of the Observed Free-Electron-Like State in a PTCDA Monolayer on Ag(111). New J. Phys. 2010, 12, 063014.

(62) Zaitsev, N. L.; Nechaev, I. A.; Chulkov, E. V. Change in Surface States of $\operatorname{Ag}(111)$ 
Thin Films Upon Adsorption of a Monolayer of PTCDA Organic Molecules. J. Exp. Theor. Phys. 2010, 110, 114-20.

(63) Marks, M.; Zaitsev, N. L.; Schmidt, B.; Schwalb, C. H.; Schöll, A.; Nechaev, I. A.; Echenique, P. M.; Chulkov, E. V.; Höfer, U. Energy shift and wave function overlap of metal-organic interface states. Phys. Rev. B 2011, 84, 081301.

(64) Zaitsev, N. L.; Nechaev, I. A.; Echenique, P. M.; Chulkov, E. V. Transformation of the $\operatorname{Ag}(111)$ Surface State Due to Molecule-Surface Interaction with Ordered Organic Molecular Monolayers. Phys. Rev. B 2012, 85, 115301.

(65) Tsirkin, S. S.; Zaitsev, N. L.; Nechaev, I. A.; Tonner, R.; Höfer, U.; Chulkov, E. V. Inelastic decay of electrons in Shockley-type metal-organic interface states. Phys. Rev. B 2015, 92, 235434.

(66) Jakob, P.; Zaitsev, N. L.; Namgalies, A.; Tonner, R.; Nechaev, I. A.; Tautz, F. S.; Höfer, U.; Sanchez-Portal, D. Adsorption geometry and the interface states: Relaxed and compressed phases of NTCDA/Ag(111). Phys. Rev. B 2016, 94, 125436.

(67) Tamai, A.; Seitsonen, A. P.; Baumberger, F.; Hengsberger, M.; Shen, Z. X.; Greber, T.; Osterwalder, J. Electronic Structure at the $\mathrm{C}_{60}$ /Metal Interface: An Angle-Resolved Photoemission and First-Principles Study. Phys. Rev. B 2008, 77, 075134.

(68) Yamane, H.; Kanai, K.; Ouchi, Y.; Ueno, N.; Seki, K. Impact of Interface Geometric Structure on Organic-Metal Interface Energetics and Subsequent Films Electronic Structure. J. Electron Spectrosc. 2009, 174, 28-34.

(69) Scheybal, A.; Müller, K.; Bertschinger, R.; Wahl, M.; Bendounan, A.; Aebi, P.; Jung, T. A. Modification of the $\mathrm{Cu}(110)$ Shockley Surface State by an Adsorbed Pentacene Monolayer. Phys. Rev. B 2009, 79, 115406. 
(70) Ziroff, J.; Gold, P.; Bendounan, A.; Forster, F.; Reinert, F. Adsorption Energy and Geometry of Physisorbed Organic Molecules on $\mathrm{Au}(111)$ Probed by Surface-State Photoemission. Surf. Sci. 2009, 603, 354-58.

(71) Andrews, K. M.; Pearl, T. P. Modification of Ag(111) Surface Electronic Structure Via Weak Molecular Adsorption of Adenine Measured with Low Temperature Scanning Tunneling Microscopy and Spectroscopy. J. Chem. Phys. 2010, 132, 214701.

(72) Heinrich, B. W.; Limot, L.; Rastei, M. V.; Iacovita, C.; Bucher, J. P.; Djimbi, D. M.; Massobrio, C.; Boero, M. Dispersion and Localization of Electronic States at a Ferrocene/Cu(111) Interface. Phys. Rev. Lett. 2011, 107, 216801.

(73) Schmid, M.; Zirzlmeier, J.; Steinrück, H. P.; Gottfried, J. M. Interfacial Interactions of Iron(II) Tetrapyrrole Complexes on $\mathrm{Au}(111)$. J. Phys. Chem. C 2011, 115, 1702817035 .

(74) Hong, S. Y.; Yeh, P. C.; Dadap, J. I.; Osgood, R. M. Interfacial Dipole Formation and Surface-Electron Confinement in Low-Coverage Self-Assembled Thiol Layers: Thiophenol and p-Fluorothiophenol on $\mathrm{Cu}(111)$. Acs Nano 2012, 6, 10622-10631.

(75) Faraggi, M. N.; Jiang, N.; Gonzalez-Lakunza, N.; Langner, A.; Stepanow, S.; Kern, K.; Arnau, A. Bonding and Charge Transfer in Metal-Organic Coordination Networks on $\mathrm{Au}(111)$ with Strong Acceptor Molecules. J. Phys. Chem. C 2012, 116, 24558-24565.

(76) Wießner, M.; Lastra, N. S. R.; Ziroff, J.; Forster, F.; Puschnig, P.; Dössel, L.; Müllen, K.; Schöll, A.; Reinert, F. Different views on the electronic structure of nanoscale graphene: aromatic molecule versus quantum dot. New Journal of Physics 2012, 14, 113008.

(77) Umbach, T. R.; Fernandez-Torrente, I.; Ladenthin, J. N.; Pascual, J. I.; Franke, K. J. Enhanced charge transfer in a monolayer of the organic charge transfer complex TTFTNAP on $\mathrm{Au}(111)$. J. Phys.: Condens. Matter 2012, 24, 354003. 
(78) Ilyas, N.; Kelly, L. L.; Monti, O. L. A. Electronic structure and dynamics of quasi-2D states of vanadyl naphthalocyanine on $\mathrm{Au}(111)$. Mol. Phys. 2013, 111, 2175-2188.

(79) Heidorn, S.; Bertram, C.; Koch, J.; Boom, K.; Matthaei, F.; Safiei, A.; Henzl, J.; Morgenstern, K. Influence of Substrate Surface-Induced Defects on the Interface State between $\mathrm{NaCl}(100)$ and $\mathrm{Ag}(111)$. Journal of Physical Chemistry C 2013, 117, 1609516103.

(80) Armbrust, N.; Schiller, F.; Güdde, J.; Höfer, U. Model potential for the description of metal/organic interface states. Scientific Reports 2017, 7, 46561.

(81) Temirov, R.; Soubatch, S.; Luican, A.; Tautz, F. S. Free-Electron-Like Dispersion in an Organic Monolayer Film on a Metal Substrate. Nature 2006, 444, 350-53.

(82) Reinert, F.; Nicolay, G.; Schmidt, S.; Ehm, D.; Hüfner, S. Direct measurements of the L-gap surface states on the (111) face of noble metals by photoelectron spectroscopy. Phys. Rev. B 2001, 63, 115415.

(83) Blumenfeld, M. L.; Steele, M. P.; Monti, O. L. Near- and Far-Field Effects on Molecular Energy Level Alignment at an Organic/Electrode Interface. The Journal of Physical Chemistry Letters 2010, 1, 145-148. 